\title{
FLAT CONNECTIONS ON ORIENTED 2-MANIFOLDS
}

\author{
LISA C. JEFFREY
}

\begin{abstract}
This paper aims to provide a survey on the subject of representations of fundamental groups of 2-manifolds, or in other guises flat connections on orientable 2-manifolds or moduli spaces parametrizing holomorphic vector bundles on Riemann surfaces. It emphasizes the relationships between the different descriptions of these spaces. The final two sections of the paper outline results of the author and Kirwan on the cohomology rings of certain of the spaces described earlier (formulas for intersection numbers that were discovered by Witten (Commun. Math. Phys. 141 (1991) 153-209 and J. Geom. Phys. 9 (1992) 303-368) and given a mathematical proof by the author and Kirwan (Ann. of Math. 148 (1998) 109-196)).
\end{abstract}

\section{Introduction}

This paper aims to provide a survey on the subject of representations of fundamental groups of 2-manifolds, or in other guises flat connections on orientable 2-manifolds or moduli spaces parametrizing holomorphic vector bundles on Riemann surfaces. Sections 1 and 2 are intended to be accessible to anyone with a background corresponding to an introductory course on differentiable manifolds (at the advanced undergraduate or beginning research student level in UK universities). Sections 3 and 4 describe recent work on the topology of the spaces treated in Sections 1 and 2, and may require more background in algebraic topology (for instance, familiarity with homology theory).

The layout of the paper is as follows. Section 1.2 treats the Jacobian (which is the simplest prototype for the class of objects treated throughout the paper, corresponding to the group $\mathrm{U}(1))$. Section 1.3 treats the case corresponding to compact nonabelian Lie groups (such as $\mathrm{SU}(n)$ ). Section 1.4 treats the case where compact manifolds without boundary are replaced by compact manifolds with one boundary component (corresponding to replacing vector bundles of degree 0 with vector bundles of nonzero degree). Section 1.5 describes a number of different contexts where the objects occur in mathematics. Section 2.1 treats the general properties of these spaces, and Section 2.2 treats spaces of connections. Section 2.3 treats cohomology in the $\mathrm{U}(1)$ case, while Section 2.4 treats the general case. Sections 3 and 4 treat recent work on the topology of these spaces.

\subsection{Background material}

Let $\Sigma$ be a compact two-dimensional orientable manifold. Unless otherwise specified, 'dimension' refers here to the dimension as a real manifold. The space $\Sigma$ can be described in different ways, depending on how much structure we choose to specify.

Received 10 October 2003; revised 27 April 2004.

2000 Mathematics Subject Classification 53D30 (primary), 14D20 (secondary). 
(1) Topological description. One may form a class of topological spaces homeomorphic to $\Sigma$ by gluing together (in pairs) the edges of a polygon with $4 g$ sides. These spaces are classified by their fundamental groups (in other words, by the genus $g$, for which the Euler characteristic of the space is $2-2 g$; a space with genus $g$ is a $g$-holed torus):

$$
\pi=\pi_{1}\left(\Sigma^{g}\right)=\left\langle a_{1}, b_{1}, \ldots, a_{g}, b_{g}: \prod_{j=1}^{g} a_{j} b_{j} a_{j}^{-1} b_{j}^{-1}=1\right\rangle .
$$

The $a_{j}$ and $b_{j}$ provide a basis of $H_{1}(\Sigma)$, chosen so that their intersection numbers are

$$
a_{j} \cap b_{j}=1,
$$

and all other intersections are zero. For more details, see [27, Chapter 1].

(2) Smooth description. The objects described in (1) may be endowed with structures of smooth orientable manifolds of dimension 2 , and all smooth structures on a compact orientable 2-manifold of genus $g$ are equivalent up to diffeomorphism.

(3) Holomorphic description. The objects in (1) and (2) may be endowed with additional structure, since they may be given the structure of a complex manifold or Riemann surface. (Riemann surfaces are complex manifolds of complex dimension 1.) The collection of possible complex structures on an orientable 2-manifold of genus $g$ is a complex variety called the moduli space of Riemann surfaces of genus $g$. For genus $g=1$, the collection of complex structures on the 2-torus is the quotient of the upper half-plane $\mathbb{H}$ by the natural action of $\mathrm{SL}(2, \mathbb{Z})$,

$$
\left[\begin{array}{ll}
a & b \\
c & d
\end{array}\right]: z \mapsto \frac{a z+b}{c z+d}
$$

The complex structure on any orientable 2-manifold of genus $g=1$ is specified by the quotient of $\mathbb{C}$ by the lattice generated by 1 and $\tau$, where $\tau \in \mathbb{H}$. The collection of complex structures on an orientable 2 -manifold of genus $g \geqslant 2$ is parametrized by the points in the quotient of $\mathbb{C}^{3 g-3}$ by the action of a discrete group.

Often, compact Riemann surfaces are described by algebraic equations in the complex projective plane $\mathbb{C} P^{2}$. For example, Riemann surfaces of genus $g=1$ may be specified by an equation

$$
\left\{(z, w): z^{2}=(w-1)(w+1)(w-\lambda)\right\}
$$

on $\mathbb{C} P^{2}$ for some $\lambda \in \mathbb{C}$.

\subsection{The Jacobian}

One may associate certain spaces with $\Sigma$ that admit different descriptions, depending on the amount of structure with which we have equipped our 2-manifold. In algebraic geometry, the moduli problem refers to the problem of describing the parameters on which a collection of algebraic varieties depend. The term moduli space originated in algebraic geometry, where it refers to a space that parametrizes a family of objects. For a detailed survey on the moduli problem, see [25]. By an abuse of language, the term 'moduli space' is often also used to refer to the same objects as they arise in other contexts where the structure of algebraic variety is not specified. Strictly speaking, this usage is incorrect, but one frequently encounters it. 
A prototype is the Jacobian, which can be described in several different ways.

(1)Topological description. If we view $\Sigma$ as a topological space and retain only the structure of its fundamental group $\pi$, we may define

$$
\operatorname{Jac}(\Sigma)=\operatorname{Hom}(\pi, \mathrm{U}(1))=\mathrm{U}(1)^{2 g} .
$$

(2) Smooth description. If we view $\Sigma$ as a smooth, orientable 2-manifold, the Jacobian has a gauge theory description

$$
\operatorname{Jac}(\Sigma)=\text { flat } \mathrm{U}(1) \text { connections/gauge group. }
$$

The space $\mathcal{A}$ of $\mathrm{U}(1)$ connections is the space $\Omega^{1}(\Sigma)$ of 1 -forms on $\Sigma$. In terms of local coordinates $y_{1}$ and $y_{2}$ on a neighbourhood $U$ in $\Sigma, \mathcal{A}$ is identified with two copies of $C^{\infty}(U)$ :

$$
\mathcal{A}=\left\{A=A_{1} d y_{1}+A_{2} d y_{2}: A_{1}, A_{2} \in C^{\infty}(U)\right\} .
$$

See Section 2.1 for a more general description of the space of connections and the gauge group, and an explanation of how the gauge group acts on the space of connections.

We can impose the condition that a connection $A$ be flat: this leads to the space $\mathcal{A}_{F}$, defined in terms of local coordinates as follows.

$$
\mathcal{A}_{F}=\left\{A=A_{1} d y_{1}+A_{2} d y_{2}: \frac{\partial A_{1}}{\partial y_{2}}-\frac{\partial A_{2}}{\partial y_{1}}=0\right\} .
$$

The gauge group is $\mathcal{G}=C^{\infty}(\Sigma, \mathrm{U}(1))$; its Lie algebra is $\operatorname{Lie}(\mathcal{G})=C^{\infty}(\Sigma)$. The space of connections is given a more detailed treatment in Section 2.2, while the present example will be developed in greater detail in Section 2.3.

(3) Holomorphic description. If we endow $\Sigma$ with a complex $\operatorname{structure,} \operatorname{Jac}(\Sigma)$ is identified with an algebraic variety that classifies holomorphic line bundles over $\Sigma$; this is how the Jacobian arises naturally in algebraic geometry. Strictly speaking, the term 'Jacobian' refers only to the algebraic-geometric object, which is a complex torus.

\subsection{Representations into a nonabelian Lie group}

When we replace $\mathrm{U}(1)$ by a compact nonabelian group $G$ (for example, $G=$ $\mathrm{SU}(2)$, or more generally $G=\mathrm{SU}(n))$, some complications arise.

(1) Topological description. The natural generalization of $\operatorname{Jac}(\Sigma)$ is

$$
\mathcal{M}(\Sigma)=\operatorname{Hom}(\pi, G) / G
$$

where $G$ acts on $\operatorname{Hom}(\pi, G)$ by conjugation.

(2) Smooth description. $\mathcal{M}(\Sigma)$ has a natural gauge theory description that generalizes the description of the Jacobian:

$$
\mathcal{M}(\Sigma) \cong \text { flat } G \text { connections on } \Sigma / \mathcal{G}
$$

where $\mathcal{G}=C^{\infty}(\Sigma, G)$ is the gauge group. 
(3) Holomorphic description. $\mathcal{M}(\Sigma)$ also has a description in algebraic geometry: it is the moduli space of holomorphic $G^{\mathbb{C}}$ bundles over $\Sigma$, with an appropriate stability condition from geometric invariant theory. The identification between the space of representations and that of holomorphic vector bundles was established by Narasimhan and Seshadri [32]. For a more detailed treatment of the algebrogeometric aspects of these moduli spaces of vector bundles, see Thaddeus' survey [36] and [31, Chapter 5, Appendix C]. See also [22-24] for some of Kirwan's results on moduli spaces of vector bundles. See [25] for a survey on moduli problems in algebraic geometry.

EXAmPle 1.1. For $G=\mathrm{U}(n), \mathcal{M}(\Sigma)$ is identified with the moduli space of (semistable) holomorphic vector bundles of rank $n$ and degree 0 over $\Sigma$. When $G=\mathrm{SU}(n)$, we obtain the moduli space of (semistable) holomorphic vector bundles of rank $n$ with trivial determinant line bundle over $\Sigma$.

\subsection{Moduli spaces of vector bundles of nonzero degree}

More generally, we can consider the moduli spaces $M(n, d)$ of (semistable) holomorphic vector bundles of rank $n$ and (possibly nonzero) degree $d$ with fixed determinant line bundle $L$ over $\Sigma$. Replace $\pi$ by

$$
\pi^{\prime}=\pi_{1}(\Sigma \backslash D)=\left\{x_{1}, \ldots, x_{2 g}\right\}
$$

(the free group on $2 g$ generators), where $D$ is a small disc in $\Sigma$. The group $\pi^{\prime}$ is the fundamental group of a compact orientable 2-manifold with one boundary component, which may be obtained by removing $D$ from $\Sigma$.

For $G=\mathrm{SU}(n)$, choose $c=e^{2 \pi i d / n} \mathbb{I}$ which generates the centre $Z(G)$ (in other words, $n$ and $d$ are coprime). For example, when $G=\mathrm{SU}(2)$ we choose $c=-\mathbb{I}$.

(1) Topological description.

$$
M(n, d)=\left\{\rho \in \operatorname{Hom}\left(\pi^{\prime}, G\right): \rho\left(\prod_{j=1}^{g} x_{2 j-1} x_{2 j} x_{2 j-1}^{-1} x_{2 j}^{-1}\right)=c\right\} / G,
$$

where $G$ acts by conjugation.

(2) Smooth description. $M(n, d)$ has a description as the space of gauge equivalence classes of flat $G$ connections on $\Sigma \backslash D$, whose holonomy around the boundary of $D$ is conjugate to $e^{2 \pi i d / n} \mathbb{I}$.

(3) Holomorphic description. $M(n, d)$ is the moduli space of (semistable) holomorphic vector bundles of rank $n$, degree $d$ and fixed determinant over $\Sigma$ (see [32]). Provided that $n$ and $d$ are coprime, the space $M(n, d)$ is a smooth manifold with a symplectic form (a closed nondegenerate 2 -form $\omega \in \Omega^{2}(M(n, d))$ - see [29] for more on symplectic geometry). The space $M(n, d)$ is also a complex manifold, and the complex structure is compatible with the symplectic form. In other words, $M(n, d)$ is a Kähler manifold.

This material will be treated in more detail in Example 2.5 of Section 2.1. 


\subsection{Importance of these spaces}

Spaces of flat connections on oriented 2-manifolds arise in a number of different contexts, as follows.

(1) Gauge theory. The properties of these spaces are a prototype for properties of topological spaces arising in gauge theory related to manifolds of dimension higher than 2 (for example Donaldson or Seiberg-Witten invariants in dimension 4 $[\mathbf{1 1}, \mathbf{3 0}]$ and Floer homology in dimension $3[\mathbf{1 6}])$.

(2) Topology. These spaces provide a natural setting for various questions involving the topology of manifolds of dimension 2 and 3. For example, the Casson invariant is an invariant of 3-manifolds; it arises naturally as the intersection number of two Lagrangian submanifolds in a space of flat connections. See, for instance, $[\mathbf{9}]$.

(3) Mathematical physics. These spaces arise from the study of the Yang-Mills equations on a manifold of dimension 2. Many topics of recent interest in quantum field theory are related to them (for example, Chern-Simons gauge theory; see [38]).

(4) Algebraic geometry. Moduli spaces of holomorphic vector bundles on Riemann surfaces have surprising properties in the context of algebraic geometry. For example, the Verlinde formula is a formula for the dimension of the space of holomorphic sections of a line bundle $\mathcal{L}$ over $M(n, d)$; see, for instance, $[\mathbf{5}-\mathbf{8}, \mathbf{1 8}]$ for more on this formula. By work of Drezet and Narasimhan [12], all holomorphic line bundles $\mathcal{L}$ arise as powers $\mathcal{L}=\mathcal{L}_{0}^{k}$ of a generating line bundle $\mathcal{L}_{0}$ over $M(n, d)$. The Verlinde formula is remarkable, since it is often difficult to determine explicitly the number of holomorphic sections of a bundle over a complex manifold.

(5) Symplectic geometry. Spaces of flat connections on oriented 2-manifolds are symplectic manifolds, and may be studied from that point of view; see, for instance, [29] for more on symplectic geometry, and [4] for symplectic geometric aspects of this example.

(6) Relation to symplectic and geometric invariant theory quotients. Atiyah and Bott [4] exhibited the spaces $M(n, d)$ as symplectic quotients via an infinitedimensional construction; the space of all connections $\mathcal{A}$ on $\Sigma$ is acted on by the gauge group $\mathcal{G}$ with moment map the curvature

$$
\mu: A \mapsto F_{A} .
$$

(See Section 2.2 for a more detailed description of connections and curvature.) Thus the symplectic quotient (in other words, $\mu^{-1}(0) / \mathcal{G}$ ) is the space of flat connections up to equivalence under the action of the gauge group. These spaces are interesting examples of quotient constructions in symplectic geometry and geometric invariant theory. It has been established $[\mathbf{4}, \mathbf{1 5}, \mathbf{2 0}, \mathbf{2 1}, \mathbf{3 1}, \mathbf{3 3}]$ that the symplectic quotient of a Kähler manifold by a (compact) group $G$ is equivalent to the geometric invariant theory quotient by the complexification $G^{\mathbb{C}}$. See, for instance, $[\mathbf{3 1}$, Chapter 8.2, Theorem 8.3] for details, and [31, Chapter 8.9] for a discussion of the relation between the symplectic and geometric invariant theory approaches to moduli spaces of vector bundles. 
2. Spaces of flat connections on oriented 2-manifolds

\subsection{General properties}

The objects of interest are the flat connections on $\Sigma$. A connection specifies a way to do parallel transport in a principal bundle over $\Sigma$ with structure group $G$. (See [26, Chapter II] for more on connections and parallel transport; see (19) below for the case $G=\mathrm{U}(1)$.) If the bundle can be trivialized, it is equivalent to the product bundle $\Sigma \times G$. There are many different ways to specify the trivialization. After one fixed choice of trivialization has been made, the choice of another trivialization is equivalent to the choice of an element of the gauge group

$$
\mathcal{G}=C^{\infty}(\Sigma, G)
$$

the group of (smooth) maps from $\Sigma$ to $G$.

The Lie algebra of $\mathcal{G}$ is the space of smooth maps from $\Sigma$ to $\operatorname{Lie}(G)$. The gauge group acts on the space of connections in the following way: if $G$ is a matrix group, a choice of a trivialization of the principal bundle identifies the space $\mathcal{A}$ of connections with $\Omega^{1}(\Sigma) \otimes \operatorname{Lie}(G)$, and an element $\Gamma \in \mathcal{G}$ sends $A \in \mathcal{A}$ to $\Gamma^{-1} A \Gamma+\Gamma^{-1} d \Gamma$.

For any closed loop $\gamma$ in $\Sigma$, a connection determines a holonomy, which is the group element $h$ such that the image of parallel transport around $\gamma$ starting at a point $\tilde{\gamma}(0)$ in the fiber above $\gamma(0)$ is obtained by multiplying $\tilde{\gamma}(0)$ by $h$. If the connection is flat (that is, the curvature $F_{A}$ is zero), then the parallel transport is not changed by continuous deformations of the loop (as long as these deformations keep the beginning point of the loop fixed). It depends only on the class of the loop as an element of the fundamental group $\pi$ (equivalence classes of loops under deformation). In fact, the action of the gauge group takes the subspace of flat connections to itself. It is not hard to deduce the following theorem.

THEOREM 2.1. The following statement holds:

$\{$ flat connections/gauge group $\} \cong\{$ representations of $\pi$ into $G\} /$ conjugation.

Sketch proof. Let $\mathcal{A}_{F}$ be the space of flat connections, and let $\mathcal{R}$ be the space of representations of $\pi$ into $G$. We define a map $\Psi$ from $\mathcal{A}_{F}$ to $\mathcal{R}$ by sending a flat connection $A$ to the representation $\rho: \pi \rightarrow G$ determined by the holonomy of $A$. We define a map $\Phi$ from $\mathcal{R}$ to $\mathcal{A}_{F}$ as follows. A representation $\rho$ of $\pi$ determines a principal $G$ bundle over $\Sigma$ with a distinguished flat connection in the following way. Let $\tilde{\Sigma}$ be the universal cover of $\Sigma$. The product bundle $\tilde{\Sigma} \times G$ over $\tilde{\Sigma}$ is equipped with an action of $\pi$ as follows: an element $\sigma \in \pi$ acts by

$$
\sigma:(x, h) \mapsto\left(x \sigma, \rho(\sigma)^{-1} h\right) .
$$

This action preserves the product connection on $\tilde{\Sigma} \times G$, so when we take the quotient by the action of $\pi$, we obtain a principal bundle $P$ over $\Sigma$ equipped with a flat connection $A(\rho)$ that comes from the product connection on $\tilde{\Sigma} \times G$. We define $\Psi(\rho)=A(\rho)$. It can be shown that $\Phi$ and $\Psi$ descend to maps between $\mathcal{A}_{F} / \mathcal{G}$ and $\mathcal{R} /\{$ conjugation $\}$, which are the inverses of each other.

The space described in Theorem 2.1 may be called the space $\mathcal{M}$ of flat connections modulo gauge transformations. According to Theorem 2.1, this is the same as the 
space of representations of the fundamental group of $\Sigma$ modulo conjugation. Let us consider some special cases of the space of representations of the fundamental group of $\Sigma$.

EXAMPle 2.1. The 2-sphere $S^{2}$.

The fundamental group of the 2-sphere is trivial (any closed loop can be shrunk to a point). Hence the space of representations is also trivial.

EXAMPLE 2.2. The torus $S^{1} \times S^{1}$.

The fundamental group of the torus is generated by two loops $a$ and $b$, and they commute with each other. Thus the fundamental group is commutative.

More generally, an oriented 2-manifold of genus $g$ ( $g$-holed torus) is formed by taking a polygon with $4 g$ sides and gluing the sides together in pairs according to the prescription given by (11) below. The sides of the polygon become the generators of the group.

Now, however, the group is not commutative: from the information that the loop around the outside of the polygon can be shrunk to a point, we learn only that the generators satisfy the relation

$$
a_{1} b_{1}\left(a_{1}\right)^{-1}\left(b_{1}\right)^{-1} \ldots a_{g} b_{g}\left(a_{g}\right)^{-1}\left(b_{g}\right)^{-1}=1 .
$$

In order to specify a representation $\rho$ of $\pi$ into a compact Lie group $G$, we must specify the elements $A_{i}$ and $B_{i}$ in $G$ to which $\rho$ sends each loop $a_{i}$ or $b_{i}$. In order that it should be a representation, we insist that the relation is preserved:

$$
A_{1} B_{1}\left(A_{1}\right)^{-1}\left(B_{1}\right)^{-1} \ldots A_{g} B_{g}\left(A_{g}\right)^{-1}\left(B_{g}\right)^{-1}=1 .
$$

We must also take the quotient by the action of $G$ by conjugation on the space of representations:

$$
h \in G: A_{i} \mapsto h^{-1} A_{i} h ; B_{i} \mapsto h^{-1} B_{i} h
$$

EXAmple 2.3. $\Sigma=S^{1} \times S^{1}, G=\mathrm{U}(n)$ (a nonabelian group).

In this case, if we choose elements $A$ and $B$ in $G$ to represent the two loops $a$ and $b$ in $S^{1} \times S^{1}$, we need to insist that

$$
A B=B A
$$

(because $a b=b a$ in $\pi$ ). Every element of $G$ is conjugate to a diagonal matrix with unit complex numbers $e^{i \theta}$ along the diagonal (that is, it can be diagonalized). If we have diagonalized $A$, and if $A$ is a generic element (in other words, if it has distinct eigenvalues), then the only elements $B$ that commute with it are the diagonal matrices with unit complex number entries. Call the space of such matrices $T$. Since we are interested in representations up to conjugation by $G$, we may assume that $A \in T$.

If $A$ and $B$ are both in $T$, we may ask what is left over of the conjugation action (in other words, what elements of $G$ will conjugate $T$ into itself?). In general, the elements of $G$ that will do this act via a finite group isomorphic to the permutation group $S_{n}$ on $n$ letters, which acts by permuting the diagonal entries. This is the Weyl group $W=N(T) / T$. So we find that

$$
\mathcal{M}=(T \times T) / W
$$


We have not described here what happens when $A$ or $B$ is not generic, but the description given in (14) remains valid. More generally, the points where $A$ and $B$ are not generic may correspond to singular points in the space of representations.

EXAMPLE 2.4. $\quad G=\mathrm{U}(1)$, the circle group.

Note that this group is commutative, so the conjugation action is the identity map

$$
A_{i} \mapsto A_{i}, B_{i} \mapsto B_{i}
$$

for any $g \in G$. Also, any elements $A_{i}, B_{i}$ of $G$ automatically satisfy the relation (12) because $A_{i} B_{i}\left(A_{i}\right)^{-1}\left(B_{i}\right)^{-1}=1$ for any $A_{i}$ and $B_{i}$.

So for this group, the space $\mathcal{M}$ is simply $\mathrm{U}(1)^{2 g}$.

EXAMPLE 2.5. The general case.

Let $G=\mathrm{U}(n)$, and suppose that $\Sigma$ is an orientable 2-manifold with genus $g>1$. In the case when $G$ is a nonabelian group (such as $\mathrm{U}(n)$ when $n>1$ ), the space is more complicated than when $G$ is abelian. Two differences are apparent. First, the relation between the images of the generators (imposed by the fact that the loop around the boundary of the polyhedron can be shrunk to a point) is no longer automatically satisfied. Secondly, the action of the group on the space of representations by conjugation is now nontrivial. In this case, the space $\mathcal{M}$ is usually not smooth. We may replace it by a smooth analogue, obtained by cutting out a small disc $D$ in $\Sigma$ and requiring that the representation send the loop around the boundary of the disc, not to 1 but to the product of the identity matrix and a root of unity $e^{2 \pi i d / n}$ that generates the $n$th roots of unity. This space (denoted $M(n, d))$ is in fact smooth, and shares many properties with the more natural space $\mathcal{M}$; we have described some of its properties in Section 1.4.

\subsection{Connections}

The space $\mathcal{A}$ of all connections is simply the vector space of 1 -forms tensored with $\operatorname{Lie}(G)$. To determine the tangent space to the space of flat connections, we use the fact that the curvature is the quantity

$$
F_{A}=d A+\frac{1}{2}[A, A] .
$$

Infinitesimally, if $F_{A}=0$, then the condition that $F_{A+a}=0$ translates to

$$
d a+[A, a]=0
$$

We write this as

$$
d_{A} a=0 .
$$

It turns out that one can generalize this definition (in a way that can easily be described) to give an operator $d_{A}$ that maps $\operatorname{Lie}(G)$-valued differential forms of degree $p$ to $\operatorname{Lie}(G)$-valued differential forms of degree $p+1$ and satisfies

$$
d_{A} \circ d_{A}=0 .
$$

At the infinitesimal level, the image of the $\operatorname{Lie}(G)$-valued 0 -forms under $d_{A}$ is the tangent space to the orbits of the group of gauge transformations. Thus the tangent 
space to the space $\mathcal{M}$ is the space

$$
\begin{aligned}
T_{A} \mathcal{M} & =H^{1}\left(\Sigma, d_{A}\right) \\
& =\frac{\left\{a \in \Omega^{1}(\Sigma) \otimes \operatorname{Lie}(G) \mid d_{A} a=0\right\}}{\left\{d_{A} \phi \mid \phi \in \Omega^{0}(\Sigma) \otimes \operatorname{Lie}(G)\right\}} .
\end{aligned}
$$

We can see from this that $\mathcal{M}$ has a symplectic form, a closed 2 -form that induces a nondegenerate skew-symmetric pairing on each tangent space. At the level of the vector space $\mathcal{A}$ of all connections, this just comes from the wedge product on differential forms, combined with an Ad-invariant inner product $\langle$,$\rangle on \operatorname{Lie}(G)$; choosing a basis $\left\{e_{\alpha}\right\}$ for $\operatorname{Lie}(G)$ we have

$$
\omega(A, B)=\sum_{\alpha, \beta}\left\langle e_{\alpha}, e_{\beta}\right\rangle \int_{\Sigma} A^{\alpha} \wedge B^{\beta} .
$$

Here we have written $A=\sum_{\alpha} A^{\alpha} e_{\alpha}$ for 1 -forms $A^{\alpha}$ (and similarly for $B$ ). One can see (using Stokes' theorem) that $\omega$ descends to a skew-symmetric pairing on $H^{1}\left(\Sigma, d_{A}\right)$ (because we have $\omega\left(d_{A} \phi, b\right)=0$ for all $b \in \Omega^{1}(\Sigma, \operatorname{Lie}(G))$ and any $\left.\phi \in \Omega^{0}(\Sigma, \operatorname{Lie}(G))\right)$. In fact, this pairing is nondegenerate. If $G$ is abelian, this pairing is just the cup product on cohomology, because in de Rham cohomology the cup product is represented by the wedge product of differential forms. This specifies a symplectic structure on $\mathcal{M}$. For more details on the construction of this symplectic structure, see $[\mathbf{1 3}, \mathbf{1 9}, \mathbf{3 7}]$.

\subsection{Cohomology of $\mathrm{U}(1)$ spaces}

Let us revisit the space $\mathcal{M}$, regarded as flat connections modulo gauge transformations in the case when $G=\mathrm{U}(1)$. A connection $A$ is simply a 1-form $\sum_{i=1}^{2} A_{i} d x^{i}$ on $\Sigma$. The holonomy of $A$ around a cycle $\gamma(t)$ in $\Sigma$ is

$$
\exp \left(i \int_{0}^{2 \pi} \sum_{j=1}^{2} A_{j}(\gamma(t)) \frac{d \gamma^{j}}{d t} d t\right),
$$

since the parallel transport $x(\cdot)$ satisfies the equation

$$
\frac{d x}{d t}=i A(\gamma(t)) x(t) .
$$

The solution to this equation is obtained by exponentiating the line integral of $A$ along $\gamma$ :

$$
x(t)=\exp \left(i \int_{\gamma(0)}^{\gamma(t)} A\left(\frac{d \gamma}{d t^{\prime}}\right) d t^{\prime}\right) .
$$

The connection $A$ is flat if and only if $d A=0$ in terms of the exterior differential $d$ (which sends $p$-forms to $(p+1)$-forms). The connection resulting from the action of an infinitesimal gauge transformation $\phi$ (where $\phi$ is a $\mathbb{R}$-valued function on $\Sigma$ ) is the 1 -form $d \phi$. Thus, when we take the quotient of the flat connections by those that arise as the image of infinitesimal gauge transformations, we obtain

$$
\frac{\{A \mid d A=0\}}{\{d \phi\}} .
$$

In de Rham cohomology, this gives the first cohomology $H^{1}(\Sigma ; \mathbb{R})$ of $\Sigma$, which is the tangent space to $\mathrm{U}(1)^{2 g}$ at the equivalence class $[A]$ containing the flat connection $A$. 
The first cohomology of $\mathrm{U}(1)^{2 g}$ is the direct sum of $2 g$ copies of the first cohomology group of $\mathrm{U}(1)$ :

$$
H^{1}(\Sigma ; \mathbb{R}) \cong \mathbb{R}^{2 g}
$$

Notice that a special case of the assertion made in Section 2.1 has an easy proof using Stokes's theorem: the parallel transport of a U(1) connection around a closed loop is independent of homotopies of the loop, and so parallel transport gives a map from the space of flat $\mathrm{U}(1)$ connections to the representations of the fundamental group. It also shows that parallel transport by a flat connection is independent of infinitesimal gauge transformations (since infinitesimal gauge transformations can be implemented by adding an exact form $d \phi$ to the connection form $A$ ).

The gauge group and its Lie algebra are described in Section 1.2. An element $\phi \in \operatorname{Lie}(\mathcal{G})$ acts on $\mathcal{A}$ by

$$
\phi: A_{j} \mapsto A_{j}+\frac{\partial \phi}{\partial y_{j}}
$$

According to (21), we have

$$
\mathcal{A}_{F} / \exp \operatorname{Lie}(\mathcal{G})=\mathbb{R}^{2 g}
$$

To take the quotient by the full gauge group $\mathcal{G}$ (rather than simply by its Lie algebra), we must divide by an additional $\mathbb{Z}^{2 g}$. (The reason we must divide by $\mathbb{Z}^{2 g}$ follows from a simple obstruction theory argument, using the fact that the only obstruction to lifting a map from $\Sigma$ to $G$ to a map from $\Sigma$ to $\operatorname{Lie}(G)$ is in $H^{1}(\Sigma, \mathbb{Z})$, which is $\mathbb{Z}^{2 g}$.) We find that

$$
\mathcal{A}_{F} / \mathcal{G} \cong \mathbb{R}^{2 g} / \mathbb{Z}^{2 g} \cong(\mathrm{U}(1))^{2 g} .
$$

We recover our previous description of this space.

The generators of the cohomology come from the generators of the cohomology for $\mathrm{U}(1)$ :

$$
S^{1}=\mathrm{U}(1)=\left\{e^{i \theta}\right\},
$$

so the cohomology is generated by the 1 -form $d \theta$, which is not equal to $d f$ for any $\mathbb{R}$-valued function $f$. (Note that $\theta$ takes values in $\mathbb{R} / 2 \pi \mathbb{Z}$, rather than in $\mathbb{R}$.) There is one relation $d \theta \wedge d \theta=0$ (since there are no nonzero 2 -forms on the 1-dimensional manifold $\mathrm{U}(1))$. Thus the cohomology of the space $\mathrm{U}(1)^{2 g}$ has $2 g$ generators $d \theta_{i}$, $i=1, \ldots, 2 g$, and the only relations are that

$$
d \theta_{i} \wedge d \theta_{j}=-d \theta_{j} \wedge d \theta_{i}, \quad i, j=1, \ldots, 2 g
$$

(and in particular $d \theta_{i} \wedge d \theta_{i}=0$ ). So the cohomology is an exterior algebra on $2 g$ generators of degree 1 .

\subsection{Cohomology: the general case}

We would like to find the analogues of these generators and relations for the case of $\mathcal{M}$ when $G$ is a nonabelian group such as $\mathrm{U}(n)$ or $\mathrm{SU}(n)$.

Here, the generators of the cohomology ring are obtained as follows. There is a vector bundle $\mathcal{U}$ (the 'universal bundle') over $\mathcal{M} \times \Sigma$. The bundle $\mathcal{U}$ has a structure of holomorphic bundle over $\mathcal{M} \times \Sigma$, such that its restriction to $\{x\} \times \Sigma$ for any point $x \in \mathcal{M}$ is the holomorphic vector bundle over $\Sigma$ parametrized by the point $x$. We take a connection $A$ on $\mathcal{U}$ and decompose polynomials in its curvature $F_{A}$ (for 
example, Trace $\left.\left(F_{A}^{n}\right)\right)$ into the product of closed forms on $\Sigma$ and closed forms on $\mathcal{M}$. We then integrate these forms over cycles in $\Sigma$ (a point or 0 -cycle, the 1-cycles $a_{i}$ and $b_{i}$, or the 2-cycle given by the entire 2-manifold $\Sigma$ ) to produce closed forms on $\mathcal{M}$, which represent the generators of the cohomology ring of $\mathcal{M}$. These classes generate the cohomology of $\mathcal{M}$ under addition and multiplication. Identifying the relations between these generators is much more difficult than in the $\mathrm{U}(1)$ case.

One important cohomology class is the cohomology class of the Kähler form on $\mathcal{M}$. The Kähler class is the cohomology class

$$
[\Sigma]
$$

obtained by taking the slant product of $c_{2}(\mathcal{U})$ with the fundamental class of $\Sigma$. Another important family of classes comprises those obtained by evaluating the classes on $\mathcal{M} \times \Sigma$ at a point in $\Sigma$. For the space $M(2,1)$, the class obtained by evaluating $c_{2}(\mathcal{U})$ at a point in $\Sigma$ is often denoted $a \in H^{4}(M(2,1))$. The class in $(25)$ is often denoted $f \in H^{2}(M(2,1))$, and it is the class arising from $c_{2}(\mathcal{U})$ evaluated on the fundamental class of $\Sigma$. This class is frequently chosen as the normalization of the cohomology class of the symplectic form on $M(2,1)$; see [34] for more details. (Newstead uses the notation $\alpha$ for a multiple of $f$ and $\beta$ for a multiple of $a$. The notation $a$ and $f$ is used in [18].)

\section{Witten's formulas}

Witten $[\mathbf{3 9}, \mathbf{4 0}]$ obtained formulas for intersection numbers in the cohomology of these spaces $M(n, d)$. In particular, he obtained formulas for their symplectic volume. For $\mathrm{SU}(2)$ these formulas are as follows.

ExAmple 3.1. $n=2, d=1$; see $[\mathbf{1 0}, \mathbf{3 5}]$.

In this case, the cohomology is generated by the generators $a \in H^{4}(M(2,1))$, $f \in H^{2}(M(2,1))$ and $b_{j} \in H^{3}(M(2,1)), j=1, \ldots, 2 g$. The structure of the cohomology ring is then determined by the relations between these generators. Since the cohomology of a compact manifold satisfies Poincaré duality, these relations are determined by the intersection numbers of all monomials in the generators. One may eliminate the odd-degree generators $b_{j}$ (see [35]), so the structure of the cohomology ring can be reduced to knowing the intersection numbers of all powers of the two even-degree generators $a$ and $f$ described above:

$$
\begin{aligned}
\int_{M(2,1)} a^{j} \exp f & =\frac{(-1)^{j}}{2^{g-2} \pi^{2(g-1-j)}} \sum_{n>0} \frac{(-1)^{n+1}}{n^{2 g-2-2 j}} \\
& =\frac{(-1)^{j}}{2^{g-2} \pi^{2(g-1-j)}}\left(1-2^{2 g-3-2 j}\right) \zeta(2 g-2-2 j) .
\end{aligned}
$$

Here we have used the notation

$$
\exp f=\sum_{m \geqslant 0} \frac{f^{m}}{m !},
$$

and we use the fact that $\int_{M(2,1)} \alpha=0$ (where the integral denotes evaluation on the fundamental class of $M(2,1))$ unless the degree of $\alpha$ equals the dimension of $M(2,1)$. 
We note that the formulas for intersection numbers can be written in terms of a sum over irreducible representations of $G$; this is the form in which these formulas appeared in Witten's work.

Example 3.2. The symplectic volume of the space $\mathcal{M}$ of gauge equivalence classes of flat $G$ connections is given by the 'Witten zeta function':

$$
\int_{\mathcal{M}} \exp (f) \sim \sum_{R} \frac{1}{(\operatorname{dim} R)^{2 g-2}},
$$

where we sum over irreducible representations $R$ of $G$. In the preceding formula and the next two formulas, the symbol $\sim$ means that the left-hand side is proportional to the right-hand side by a known proportionality constant; for the details, see [39]. In the special case of $\mathrm{SU}(2)$, we have

$$
\int_{\mathcal{M}} \exp (f) \sim \sum_{n} \frac{1}{n^{2 g-2}}
$$

and

$$
\int_{M(2,1)} \exp (f) \sim \sum_{n} \frac{(-1)^{n+1}}{n^{2 g-2}},
$$

where we sum over the irreducible representations of $\mathrm{SU}(2)$, which are parametrized by their dimensions $n$. In this case, the Witten zeta function reduces to the Riemann zeta function.

The symplectic volume forms the subject of [39]. Witten expresses the volume in terms of Reidemeister-Ray-Singer torsion, and gives a mathematically rigorous argument for calculating it. He also gives several physical arguments, including one that relies on the asymptotics of the Verlinde formula, and another that refers to earlier work of Migdal [28].

\section{Mathematical proof of Witten's formulas}

The space $\mathcal{M}$ is a symplectic quotient

$$
\mu^{-1}(0) / \mathcal{G}
$$

where $\mu$ is the moment map (a collection of Hamiltonian functions whose Hamiltonian flows generate the action of a group $\mathcal{G}$ on a symplectic manifold $M$ ).

(1) The space $\mathcal{M}$ may be constructed as an infinite-dimensional symplectic quotient of the space of all connections $\mathcal{A}$ by the gauge group $\mathcal{G}$ : the moment map

$$
\mu: \mathcal{A} \rightarrow \operatorname{Lie}(\mathcal{G})^{*}
$$

of a connection $A$ is its curvature

$$
\mu(A)=F_{A} \in \Omega^{2}(\Sigma, \operatorname{Lie}(G))=\Omega^{0}(\Sigma, \operatorname{Lie}(G))^{*}
$$

(and $\Omega^{0}(\Sigma, \operatorname{Lie}(G))$ is the Lie algebra of the gauge group). Hence $\mu^{-1}(0) / \mathcal{G}$ is the space $\mathcal{M}$.

(2) The space $\mathcal{M}$ may also be constructed as a finite-dimensional symplectic quotient of a (finite-dimensional) space of flat connections on a punctured Riemann surface, by the action of the finite-dimensional group $G$. This may involve an extended moduli space $[\mathbf{1 7}]$ or the quotient of a space with a groupvalued moment map $[\mathbf{1}-\mathbf{3}]$. 
We use formulas [18] for intersection numbers in a symplectic quotient, in terms of the restriction to the fixed points of the action of a maximal commutative subgroup of $G$ (for $G=\mathrm{U}(n)$, this subgroup comprises the diagonal matrices $\mathrm{U}(1)^{n}$ ). The answer is given in terms of:

(1) the action of the maximal torus $T$ on the normal bundle to the fixed point set to the $T$ action;

(2) the values of the moment map on the fixed-point set;

(3) the restriction of the cohomology classes to the fixed-point set. Using these methods, we recover Witten's formulas.

\section{References}

1. A. Alekseev, A. Malkin and E. Meinrenken, Lie group valued moment maps, J. Differential Geom. 48 (1998) 445-495.

2. A. Alekseev, E. Meinrenken and C. Woodward, 'Group-valued equivariant localization', Invent. Math. 140 (2000) 327-350.

3. A. Alekseev, E. Meinrenken and C. Woodward, 'Duistermaat-Heckman measures and moduli spaces of flat bundles over surfaces', Geom. Funct. Anal. 12 (2002) 1-31.

4. M. F. Атіуан and R. Bотт, 'The Yang-Mills equations over Riemann surfaces', Phil. Trans. Roy. Soc. Lond. A308 (1983) 523-615.

5. A. BeAuville, 'Vector bundles on curves and generalized theta functions: recent results and open problems', Current topics in complex algebraic geometry (Berkeley, CA, 1992/93), Math. Sci. Res. Inst. Publ. 28 (Cambridge Univ. Press, 1995) 17-33.

6. A. Bertram and A. Szenes, 'Hilbert polynomials of moduli spaces of rank 2 vector bundles II', Topology 32 (1993) 599-609.

7. J.-M. Bismut and F. Labourie, 'Symplectic geometry and the Verlinde formulas', Surveys in differential geometry: differential geometry inspired by string theory, Surv. Differ. Geom. 5 (International Press, Boston, MA, 1999) 97-311.

8. R. Вотт, 'On E. Verlinde's formula in the context of stable bundles', Internat. J. Modern Phys. A 6 (1991) 2847-2858; 'Stable bundles revisited', Surv. Differ. Geom. 1 (1991) 1-18.

9. S. Cappell, R. Lee and E. Miller, 'A symplectic geometry approach to generalized Casson's invariants of 3-manifolds', Bull. Amer. Math. Soc. 22 (1990) 269-275.

10. S. K. Donaldson, 'Gluing techniques in the cohomology of moduli spaces', Topological methods in modern mathematics (Publish or Perish, 1994) 137-170.

11. S. K. Donaldson and P. Kronheimer, The geometry of four-manifolds (Oxford University Press, 1990).

12. J.-M. Drezet and M. S. Narasimhan, 'Groupe de Picard des variétés de modules de fibrés semistables sur les courbes algébriques', Invent. Math. 97 (1989) 53-94.

13. W. Goldman, 'The symplectic nature of fundamental groups of surfaces', Adv. in Math. 54 (1984) 200-225.

14. W. Goldman, 'The complex-symplectic geometry of SL(2)-characters over a Riemann surface', Volume dedicated to M. S. Raghunathan on his sixtieth birthday, 2003; available from http://www.math.umd.edu/ wmg.

15. V. Guillemin and S. SternberG, 'Geometric quantization and multiplicities of group representations', Invent. Math. 67 (1982) 515-538.

16. H. Hofer, C. H. Taubes, A. Weinstein and E. Zehnder, eds, The Floer memorial volume, Progr. Math. 133 (Birkhäuser, Basel, 1995).

17. L. Jeffrey, 'Extended moduli spaces of flat connections on Riemann surfaces', Math. Ann. 298 (1994) 667-692.

18. L. JEFFREY and F. KIRWAN, 'Intersection pairings in moduli spaces of vector bundles of arbitrary rank over a Riemann surface', Ann. of Math. 148 (1998) 109-196.

19. Y. Karshon, 'An algebraic proof for the symplectic structure of moduli space', Proc. Amer. Math. Soc. 116 (1992) 591-605.

20. G. KEMPF and L. Ness, 'The length of vectors in representation spaces', Algebraic geometry (Proc. Summer Meeting, Univ. Copenhagen, Copenhagen, 1978), Lecture Notes in Math. 732 (Springer, 1979) 233-243.

21. F. KIRwAn, Cohomology of quotients in symplectic and algebraic geometry, Math. Notes 31 (Princeton Univ. Press, 1984).

22. F. KIRWAN, 'On spaces of maps from Riemann surfaces to Grassmannians and applications to the cohomology of moduli of vector bundles', Ark. Mat. 24 (1986) 221-275.

23. F. KiRwAN, 'On the homology of compactifications of moduli spaces of vector bundles over a Riemann surface', Proc. London Math. Soc. (3) 53 (1986) 237-266. 
24. F. KIRWAN, 'The cohomology rings of moduli spaces of bundles over Riemann surfaces', J. Amer. Math. Soc. 5 (1992) 853-906.

25. F. Kirwan, 'Moduli spaces in mathematics and physics'. Proceedings of the European Women in Mathematics Workshop on Moduli Spaces (Oxford, July 1998) (ed. F. Kirwan, S. Paycha and T. S. Tsun, Hindawi, 1999) 1-16.

26. S. Kobayashi and K. Nomizu, Foundations of differential geometry, vol. I (Wiley, 1969).

27. W. S. Massey, Algebraic topology: an introduction, Grad. Texts in Math. 56 (Springer, New York, 1977).

28. A. Migdal, 'Recursion equations in gauge field theories', J. Exp. Theor. Phys. 42 (1975) 413-418.

29. D. MCDuff and D. SAlamon, Introduction to symplectic topology (Oxford Univ. Press, 1998).

30. J. Morgan, The Seiberg-Witten equations and applications to the topology of smooth fourmanifolds (Princeton Univ. Press, 1996).

31. D. Mumford, J. Fogarty and F. Kinwan, Geometric invariant theory, 3rd enlarged edn (Springer, 1994).

32. M. S. NARASimhan and C. S. Seshadri, 'Stable and unitary vector bundles on a compact Riemann surface', Ann. of Math. 82 (1965) 540-567.

33. L. NEss, 'A stratification of the null cone via the moment map', Amer. J. Math. 106 (1984) 1281.

34. P. Newstead, 'Characteristic classes of stable bundles of rank 2 over an algebraic curve', Trans. Amer. Math. Soc. 169 (1972) 337-345.

35. M. Thaddeus, 'Conformal field theory and the cohomology of the moduli space of stable bundles', J. Differential Geom. 35 (1992) 131-149.

36. M. Thaddeus, 'An introduction to the topology of the moduli space of stable bundles on a Riemann surface', Geometry and physics (Aarhus, 1995), Lecture Notes in Pure and Appl. Math. 184 (Dekker, New York, 1997) 71-99.

37. A. Weinstein, The symplectic structure on moduli space, [16] 627-635.

38. E. Witten, 'On quantum field theory and the Jones polynomial', Commun. Math. Phys. 121 (1989) 351-399.

39. E. Witten, 'On quantum gauge theories in two dimensions', Commun. Math. Phys. 141 (1991) 153-209.

40. E. Witten, 'Two-dimensional gauge theories revisited', J. Geom. Phys. 9 (1992) 303-368.

\author{
Lisa C. Jeffrey \\ Department of Mathematics \\ University of Toronto \\ Toronto M5S 3G3 \\ Canada \\ jeffrey@math.toronto.edu
}

